\title{
Individual dosimetry system for targeted alpha therapy based on PHITS coupled with microdosimetric kinetic model
}

\author{
Tatsuhiko Sato ${ }^{1,2^{*}}$ (D), Takuya Furuta ${ }^{1}$, Yuwei Liu ${ }^{3}$, Sadahiro Naka ${ }^{4}$, Shushi Nagamori ${ }^{5}$, Yoshikatsu Kanai ${ }^{6}$ and
} Tadashi Watabe ${ }^{3}$

\author{
* Correspondence: sato.tatsuhiko@ \\ jaea.go.jp \\ ${ }^{1}$ Nuclear Science and Engineering \\ Center, Japan Atomic Energy \\ Agency, Shirakata 2-4, Tokai, Ibaraki \\ 319-1195, Japan \\ ${ }^{2}$ Research Center for Nuclear \\ Physics, Osaka University, Suita, \\ Japan \\ Full list of author information is \\ available at the end of the article
}

\begin{abstract}
Background: An individual dosimetry system is essential for the evaluation of precise doses in nuclear medicine. The purpose of this study was to develop a system for calculating not only absorbed doses but also EQDX $(\alpha / \beta)$ from the PET-CT images of patients for targeted alpha therapy (TAT), considering the dose dependence of the relative biological effectiveness, the dose-rate effect, and the dose heterogeneity.
\end{abstract}

Methods: A general-purpose Monte Carlo particle transport code PHITS was employed as the dose calculation engine in the system, while the microdosimetric kinetic model was used for converting the absorbed dose to EQDX( $\alpha / \beta)$. PHITS input files for describing the geometry and source distribution of a patient are automatically created from PET-CT images, using newly developed modules of the radiotherapy package based on PHITS (RT-PHITS). We examined the performance of the system by calculating several organ doses using the PET-CT images of four healthy volunteers after injecting ${ }^{18} \mathrm{~F}-\mathrm{NKO}-035$.

Results: The deposition energy map obtained from our system seems to be a blurred image of the corresponding PET data because annihilation $\gamma$-rays deposit their energies rather far from the source location. The calculated organ doses agree with the corresponding data obtained from OLINDA 2.0 within 20\%, indicating the reliability of our developed system. Test calculations by replacing the labeled radionuclide from ${ }^{18} \mathrm{~F}$ to ${ }^{211}$ At suggest that large dose heterogeneity in a target volume is expected in TAT, resulting in a significant decrease of EQDX $(\alpha / \beta)$ for higher-activity injection.

Conclusions: As an extension of RT-PHITS, an individual dosimetry system for nuclear medicine was developed based on PHITS coupled with the microdosimetric kinetic model. It enables us to predict the therapeutic and side effects of TAT based on the clinical data largely available from conventional external radiotherapy.

Keywords: Individual dosimetry, Targeted alpha therapy, Microdosimetry, EQDX, Monte Carlo (c) The Author(s). 2021 Open Access This article is licensed under a Creative Commons Attribution 4.0 International License, which permits use, sharing, adaptation, distribution and reproduction in any medium or format, as long as you give appropriate credit to the original author(s) and the source, provide a link to the Creative Commons licence, and indicate if changes were made. The images or other third party material in this article are included in the article's Creative Commons licence, unless indicated otherwise in a credit line to the material. If material is not included in the article's Creative Commons licence and your intended use is not permitted by statutory regulation or exceeds the permitted use, you will need to obtain permission directly from the copyright holder. To view a copy of this licence, visit http://creativecommons.org/licenses/by/4.0/. 


\section{Background}

Recently, targeted alpha therapy (TAT) is gaining grounds as a novel treatment for refractory cancer, particularly after an excellent treatment effect of ${ }^{225}$ Ac-PSMA-617 [1]. We have already proved the therapeutic efficacies of $\left[{ }^{211} \mathrm{At}\right] \mathrm{NaAt}$ against differentiated thyroid cancer, ${ }^{211}$ At-labeled phenylalanine for glioma, and ${ }^{225}$ Ac-labeled fibroblast activation protein inhibitors (FAPI) against pancreatic cancer in preclinical studies [2-4]. For clinical translation, physicians initiated clinical trial is under preparation using $\left[{ }^{211} \mathrm{At}\right] \mathrm{NaAt}$ in patients with differentiated thyroid cancer refractory to radio-iodine $\left({ }^{131} \mathrm{I}\right)$ treatment. However, the TAT drugs which are successful in clinical application is still limited, and we need practical tools to evaluate the precise dose in the target and risk organs to define the most suitable dose for individual patients.

The absorbed dose (Gy) has generally been used as the primary index for predicting the therapeutic effects on tumor and unintended harmful effects on normal tissue, both in preclinical and clinical trials. In addition, higher relative biological effectiveness (RBE) must be considered in this prediction because $\alpha$ particles densely deposit their energies along their tracks and effectively induce cell killing compared to X-rays and $\beta$ particles with the same dose. For simplicity, a fixed RBE value of 5 is recommended to use in the dosimetry of TAT [5]. However, actual values of RBE intrinsically depend on the absorbed dose. Thus, explicit consideration of the dose dependence of RBE in the design of TAT is desired in the same way as the carbon ion therapy [6]. In addition, the repair mechanism during the irradiation must also be considered because of a relatively lower dose rate of TAT in comparison to external radiotherapy. Therefore, the concept of the equieffective dose, $\operatorname{EQDX}(\alpha / \beta)$, formalism was proposed to use in the TAT dosimetry [7], where EQDX represents the absorbed dose to give the same biological effect of the reference treatment, e.g., fractionated X-ray therapy [8]. The commonly used biological effective dose, $\operatorname{BED~[9],~is~a~special~case~of~} \operatorname{EQDX}(\alpha / \beta)$. Using $\operatorname{EQDX}(\alpha / \beta)$, the therapeutic and side effects of TAT can be predicted from the clinical data largely available from conventional external radiotherapy.

Dosimetry systems based on standardized phantoms such as OLINDA/EXM [10] and IDAC-Dose 2.1 [11] are widely used to estimate organ doses in nuclear medicine. However, they have some shortcomings when applied to the targeted radionuclide therapy (TRT) including TAT. For example, they cannot consider detailed anatomical differences of each patient and cannot calculate the heterogeneity of absorbed doses in the target tumor and normal tissues, which may influence tumor response and normal tissue toxicity. Therefore, several authors [12-18] developed 3-dimensional dosimetry systems by automatically creating patient-specific human phantoms and spatial distributions of radionuclides from CT and PET/SPECT images, respectively. These systems allow for a sophisticated design of TRT by calculating more detailed dosimetric quantities such as dose-mass histograms (DMH) in target tumor and normal tissues. In addition, some of them have a function of evaluating BED based on their calculated absorbed doses and dose rates. However, none of the existing system was capable of calculating $\operatorname{EQDX}(\alpha / \beta)$ for TAT, considering the complex dose dependence of RBE.

Under these situations, we developed a patient-specific dosimetry system that can calculate $\operatorname{EQDX}(\alpha / \beta)$ for TAT as well as other TRT, based on the Particle and Heavy Ion Transport code System (PHITS) [19] coupled with the microdosimetric kinetic model (MKM) [20]. The accuracy of RBE estimated by PHITS coupled with MKM was well 
verified for proton therapy [21], carbon-ion therapy [22], and boron neutron capture therapy (BNCT) [23]. In the system, a voxel phantom and a cumulative activity distribution map of a patient are automatically created in the PHITS input format from PET-CT images, respectively. After the PHITS simulation using these input files, $\operatorname{EQDX}(\alpha / \beta)$ as well as the total absorbed dose and deposition energy in each voxel are estimated, considering the microscopic dose distribution and dose rate. In this study, the performance of the system was examined using the dynamic PET-CT data, and the results were compared with corresponding data obtained from OLINDA 2.0 [10].

\section{Methods}

\section{Individual dosimetry system based on PHITS}

Figure 1 shows the flowchart of our dosimetry system, which was developed as an extension of the radiotherapy package based on PHITS, so-called RT-PHITS. It can be divided into three processes: (1) conversion from PET-CT images to PHITS input files, (2) calculation of absorbed doses using PHITS, and (3) estimation of EQDX $(\alpha / \beta)$ based on the PHITS results coupled with MKM. $\operatorname{EQDX}(\alpha / \beta)$ as well as total dose and deposition energy in each voxel are converted in DICOM RT-DOSE format. Thus, they can be imported to commercial DICOM software for further analysis. Details of each process are described below.

\section{Conversion from PET-CT images to PHITS input files}

Firstly, the patient-specific voxel phantom in the PHITS input format is created from his/her CT image using the CT2PHITS module, which was formerly called DICOM2PHITS [19]. Then, we adopted the correlation between CT numbers (Hounsfield Unit) and tissue parameters proposed by Schneider et al. [24] in this conversion, though users can define their own formula to represent the correlation in our system. The tallies for scoring the absorbed doses in Gy and deposition energies in $\mathrm{MeV}$ are also generated during this process. The resolutions of the created voxel phantom and mesh tallies are the same as the CT image.

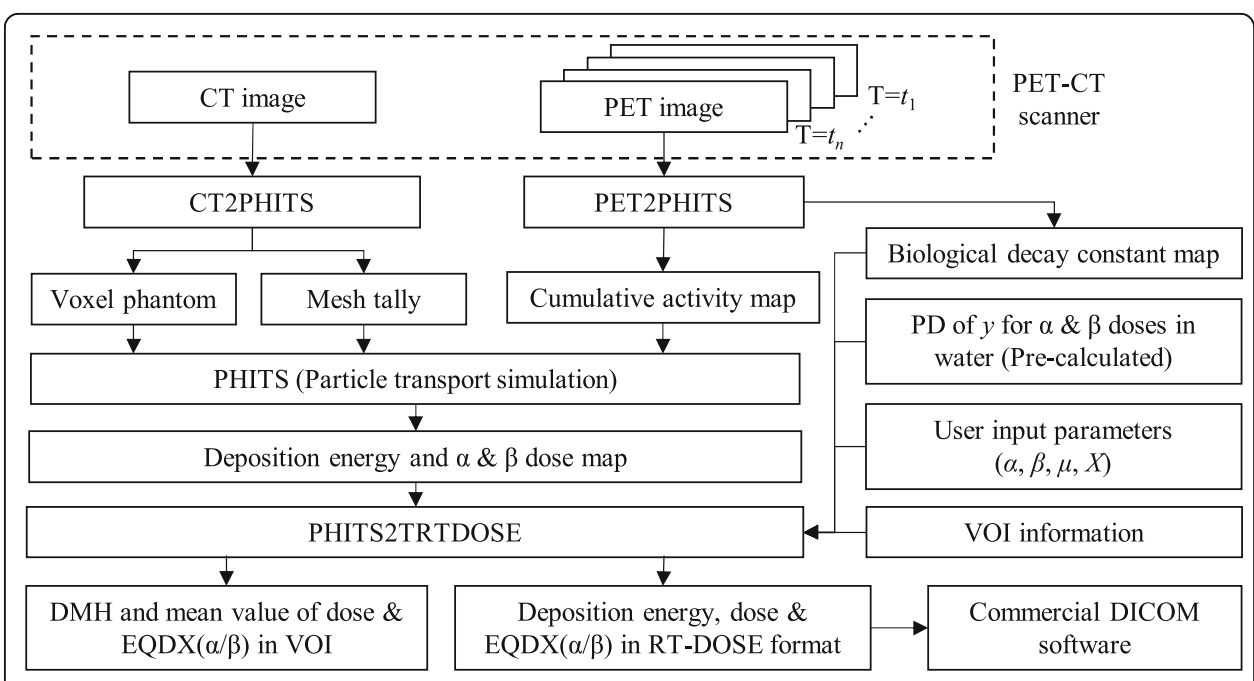

Fig. 1 Flowchart of the individual dosimetry system based on PHITS 
A new module of RT-PHITS named PET2PHITS was developed in this study to create the maps of the cumulative activities as well as biological decay constants of the radionuclides based on the PET images. There are two types of patient-specific dosimetry systems; one is to create time-dependent activity maps and execute the particle transport simulations for each time step, and the other is to create a cumulative activity map and execute a single particle transport simulation. Using the former method, dynamical dose evaluation is possible by fitting the calculated doses for each time step. However, it is very time-consuming because the Monte Carlo simulation needs to be continued until sufficiently small statistical uncertainties of the calculated doses in each voxel and time step are obtained to achieve the meaningful fitting. We therefore adopted the latter method; our system determines the cumulative activities and the biological decay constants of the radionuclides by fitting the dynamic PET images. Then, the dose rates are estimated under the assumption that they are proportional to the sum of the physical and biological decay constants of nearby voxels. The detail procedures for determining the cumulative activities and the biological decay constants are shown in Appendix A.

\section{Calculation of absorbed doses using PHITS}

Using the input files created from CT and PET images, PHITS simulation is performed to calculate the absorbed doses in the patient. In this study, PHITS version 3.20 was employed, and the EGS5 mode [25] was used for the photon, electron, and positron transport. The fluences of the source particles including the contributions from daughter nuclides are determined from the RI source generation function in PHITS, based on ICRP Publication 107 [26]. The absorbed doses due to the ionization induced by $\alpha$ and $\beta^{ \pm}$particles (referred to $\alpha$ and $\beta$ doses, respectively) were separately calculated in the simulation. Note that the kerma approximation was not adopted, and thus, the photon doses were categorized as their secondary particle doses, i.e., $\beta$ dose.

Before performing the particle transport simulation inside the patient body, another PHITS simulation must be performed to calculate the dose probability densities (PD) of lineal energy, $d(y)$, in water for $\alpha$ and $\beta$ doses, which are to be provided to MKM for the RBE estimation. The definition of the fundamental microdosimetric quantities such as lineal energy $y$ is described in Appendix B. This simulation is required once for each radionuclide because it is not specific at each patient. The microdosimetric function of PHITS [27] is utilized for this calculation because the site size of $y$ needed to be evaluated for MKM is too small (less than $1 \mu \mathrm{m}$ ) to be handled with the condensed history method employed in EGS5. Note that the microdosimetric function was developed by fitting the results of track-structure simulation. Thus, it can analytically determine the PD of $y$ down to the nanometer scales, considering the dispersion of deposition energies from the production of $\delta$-rays. Figure 2 shows examples of the calculated PD of $y$ for $\alpha$ and $\beta$ doses of ${ }^{211}$ At.

\section{Estimation of $\operatorname{EQDX}(\alpha / \beta)$}

$\operatorname{EQDX}(\alpha / \beta)$ is defined as the total absorbed dose delivered by the reference treatment plan (fraction size $X$ ) leading to the same biological effect as a test treatment plan [8]. Assuming that the biological effectiveness is proportional to the cell surviving fraction 


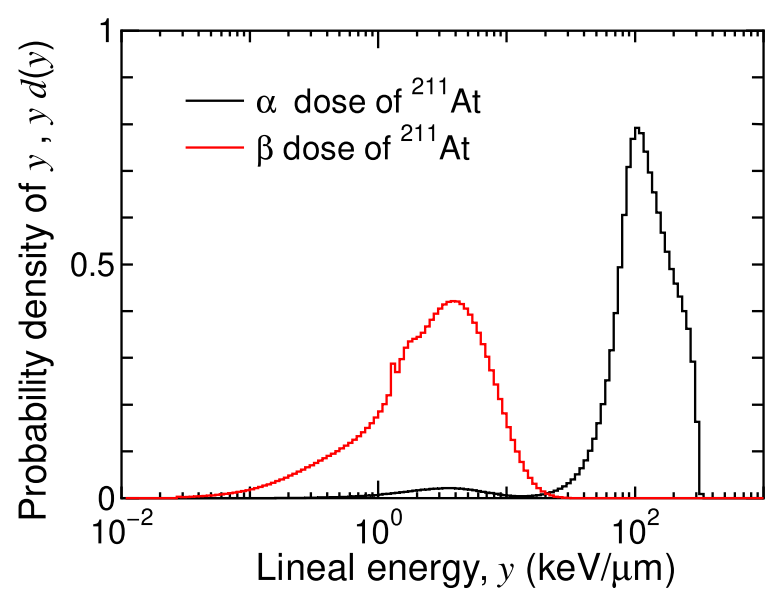

Fig. 2 Calculated dose PD of $y, y d(y)$, for $r_{d}=0.282 \mu \mathrm{m}$ for the $a$ and $\beta$ doses of ${ }^{211}$ At

following a linear-quadratic (LQ) relationship, $\operatorname{EQDX}(\alpha / \beta)$ for a test treatment with the surviving fraction $S$ can be calculated by

$$
\operatorname{EQDX}\left(\frac{\alpha}{\beta}\right)=\frac{-\ln (S)}{\alpha+\beta X},
$$

where $\alpha$ and $\beta$ are the LQ parameters for the reference treatment. Based on MKM with the extensions of the saturation correction due to the overkill effect [28] and the dose rate effect [29], the cell surviving fraction in any radiation field with an absorbed dose $D$ can be estimated by

$$
S(D)=\exp \left[-\left(\alpha_{0}+\beta z_{1 \mathrm{D}}^{*}\right) D-G \beta D^{2}\right]
$$

where $\alpha_{0}$ is the linear coefficient of the surviving fraction with the limit of LET $\rightarrow 0, G$ is the correction factor due to the dose rate effect, and $z_{1 \mathrm{D}}^{*}$ is the saturation-corrected dose-mean specific energy, deduced by

$$
z_{1 \mathrm{D}}^{*}=\frac{1}{\pi r_{\mathrm{d}}^{2}} y^{*}=\frac{1}{\pi r_{\mathrm{d}}^{2}} y_{0}^{2} \int \frac{\left[1-\exp \left(-y^{2} / y_{0}^{2}\right)\right] d(y)}{y} \mathrm{~d} y,
$$

where $y^{\prime \prime}$ is the saturation-corrected lineal energy, $r_{\mathrm{d}}$ is the radius of a subcellular structure referred to as domain, $y_{0}$ is a so-called saturation parameter that indicates the lineal energy above which the saturation correction due to the overkill effect becomes very important, and $d(y)$ is the dose probability density in domain. $d(y)$ in each voxel can be determined from its $\alpha$ and $\beta$ doses, $D_{\alpha}$ and $D_{\beta}$, respectively, as written by

$$
d(y)=\frac{D_{\alpha} d_{\alpha}(y)+D_{\beta} d_{\beta}(y)}{D_{\alpha}+D_{\beta}},
$$

where $d_{\alpha}(y)$ and $d_{\beta}(y)$ are their dose PD for each radionuclide precalculated by PHITS using the microdosimetric function. More detailed descriptions about the features of MKM are given in Appendix B in addition to the definition of fundamental microdosimetric quantities. 
Assuming that the dose rates of TRT are expressed as a mono-exponential function with a decay constant of $\lambda_{\text {phy }}+\lambda_{\text {bio }}$, where $\lambda_{\text {phy }}$ and $\lambda_{\text {bio }}$ are the physical and biological decay constants, respectively, the value of $G$ can be calculated using [13]

$$
G=\frac{\lambda_{\text {phy }}+\lambda_{\text {bio }}}{\mu+\lambda_{\text {phy }}+\lambda_{\text {bio }}}
$$

where $\mu$ is the recovery rate constant. The parameters $\alpha, \beta, \mu, \alpha_{0}, r_{\mathrm{d}}$, and $y_{0}$ depend on the cell line. Among them, $\alpha_{0}, r_{\mathrm{d}}$, and $y_{0}$ are specific to MKM, and their determination requires the experimental data of cell surviving fractions for various ion irradiations, which are generally not available. Thus, we fixed $r_{\mathrm{d}}$ and $y_{0}$ to $0.282 \mu \mathrm{m}$ and $93.4 \mathrm{keV} /$ $\mu \mathrm{m}$, respectively, which were evaluated from the surviving fractions of the HSG cell irradiated by various radiations including He ions [30,31], and calculated $\alpha_{0}$ from $\alpha$ and $z_{1 \mathrm{D}}^{*}$ for the reference radiation, $z_{1 \mathrm{D}, \text { ref }}^{*}$, using the equation of $\alpha_{0}=\alpha-\beta z_{1 \mathrm{D} \text {,ref }}^{*}$. Then, the user input parameters to our dosimetry system are $\alpha, \beta$, and $\mu$, which can be obtained from the measured surviving fractions of the reference radiation, as well as the fraction size $X$. Referring to our previous works [23, 30], we set $\alpha=0.251 \mathrm{~Gy}^{-1}, \beta=0.0615$ $\mathrm{Gy}^{-2}, \mu=1.5 \mathrm{~h}^{-1}$, and $X=2 \mathrm{~Gy}$ in the test simulations performed in this study. Consequently, $\operatorname{EQDX}(\alpha / \beta)$ calculated in this study can be expressed as EQD2(4.08), where 4.08 is the $\alpha / \beta$ ratio, i.e., $0.251 / 0.0615$.

$\operatorname{EQDX}(\alpha / \beta)$ in a certain voxel can be simply calculated from Eq. 1 by substituting the surviving fraction in the voxel obtained from Eq. 2. In contrast, special care should be taken when $\operatorname{EQDX}(\alpha / \beta)$ in a certain volume of interest (VOI) consisting of multiple voxels such as tumor and normal tissue is calculated because of the non-linear relationship between the $\operatorname{EQDX}(\alpha / \beta)$ and the surviving fraction. In such cases, the mean surviving fraction in VOI, $S_{\mathrm{VOI}}$, is given by

$$
S_{\mathrm{VOI}}=\frac{\sum_{i} S_{i}\left(D_{i}\right) m_{i}}{\sum_{i} m_{i}}
$$

where $S_{i}, D_{i}$, and $m_{i}$ are the surviving fraction, dose, and mass, respectively, of voxel $i$ made up of VOI. EQDX $(\alpha / \beta)$ in VOI can be obtained from Eq. 1 by supplying $S_{\mathrm{VOI}}$ to $S$ in similar to the concept of the equivalent uniform dose (EUD) [32]. DMH in VOI is the key quantity in this evaluation, which can be also calculated from our dosimetry system. These calculations are performed by a newly developed module of RT-PHITS named PHITS2TRTDOSE.

\section{Dynamic PET-CT acquisition and analysis}

This study was approved by the institutional review board, and written informed consents were obtained from all participants. The performance of the system was examined using the dynamic PET-CT data of four healthy volunteers after injecting ${ }^{18} \mathrm{~F}$-labeled NKO-035 with $221.6 \pm 3.8 \mathrm{MBq}$, which is a specific substrate of L-type amino acid transporter-1 (LAT1). The dynamic PET data were acquired in nine frames (total scan duration: $90 \mathrm{~min}$ ) with low-dose CT scan. All images were depicted by OSIRIX (Newton Graphics, Inc., Sapporo, Japan). Details of the data acquisition procedures were described in Appendix C. In the dose estimation, NKO-035 was assumed to be labeled with not only ${ }^{18} \mathrm{~F}$ but also ${ }^{211} \mathrm{At},{ }^{131} \mathrm{I}$, and ${ }^{177} \mathrm{Lu}$ with the same distribution in the body. Volume of interest was placed in major organs on 
dynamic PET images using PMOD software (PMOD Technologies Ltd., Zurich, Switzerland) with reference to CT images. The residence times in major organs and tissues were estimated for each patient based on their dynamic PET data using the method described in Appendix A. Supplying those data into OLINDA 2.0, the organ doses were calculated and compared with the corresponding data obtained from our dosimetry system by Bland-Altman analysis.

\section{Results}

Figure 3 shows the coronal view of CT and PET scans for a volunteer after injecting ${ }^{18} \mathrm{~F}$ NKO-035, and the corresponding deposition energy, absorbed dose, and EQD2(4.08) maps, where 4.08 is the $\alpha / \beta$ ratio. The history number of the PHITS simulation was set to 300 million so that the statistical uncertainties are very small. The deposition energy map seems to be a blurred image of the PET data particularly around the high-activity organs such as kidney and bladder because annihilation $\gamma$-rays deposit their energies rather far from the source location. In contrast, the dose and EQD2(4.08) maps exhibit higher values even at low activity regions such as the lungs. This is because the dose and EQDX $(\alpha / \beta)$ are closely related to the activity per mass (and not volume) and consequently tend to be higher at low-density regions. The relative distributions of the dose and EQD2(4.08) are similar to each other, though the absolute values of EQD2(4.08) are approximately $74 \%$ of the corresponding dose as discussed later.

Table 1 summarises the absorbed doses in the brain, lung, liver, spleen, pancreas, and kidney obtained from RT-PHITS and OLINDA 2.0. It constitutes the mean values and standard deviations of the four volunteers after injection of NKO-035 virtually labeled with $1 \mathrm{MBq}$ of ${ }^{18} \mathrm{~F},{ }^{211} \mathrm{At},{ }^{131} \mathrm{I}$, or ${ }^{177} \mathrm{Lu}$. The mean organ doses for four volunteers calculated by RT-PHITS agree with the corresponding OLINDA data mostly within $20 \%$. Figure 4 shows the Bland-Altman plot between the mean and percent difference of the organ doses calculated by RT-PHTIS and OLINDA 2.0 for each volunteer, radioisotope, and organ. It is evident that data are scattered randomly with respect to the mean organ doses.

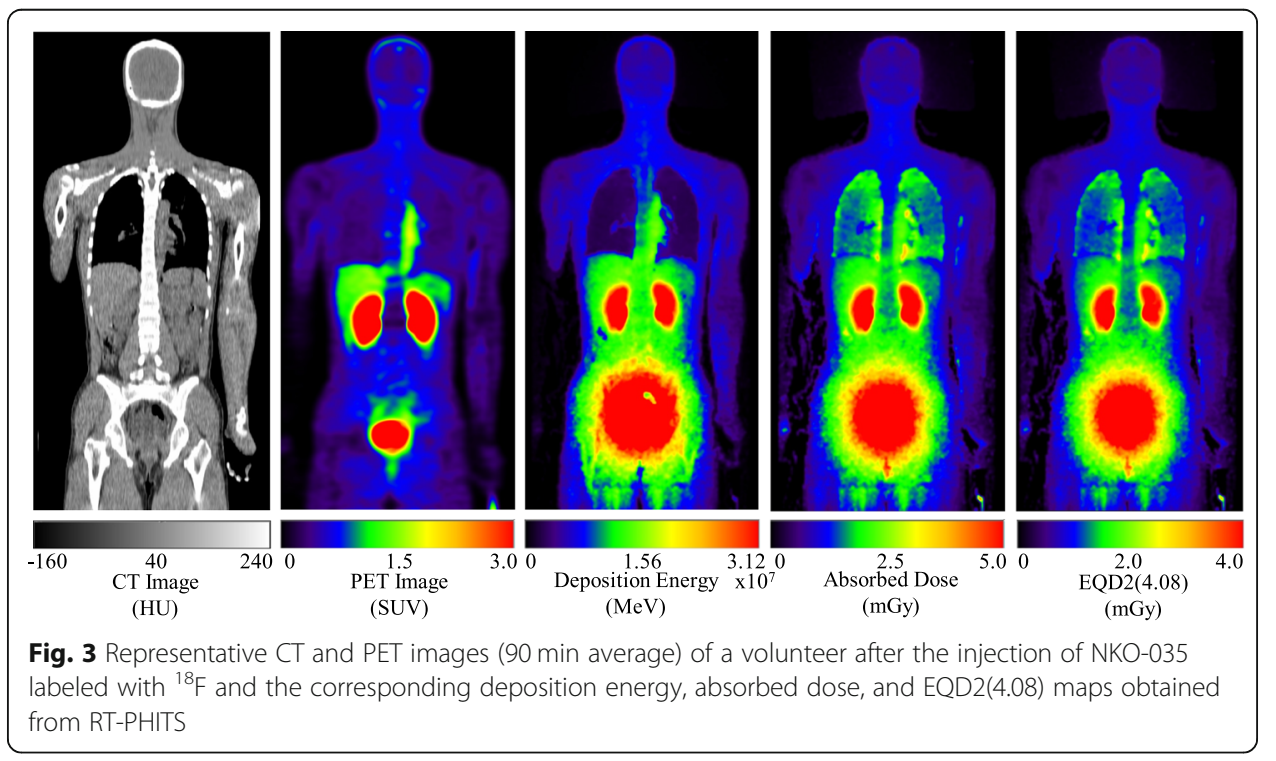




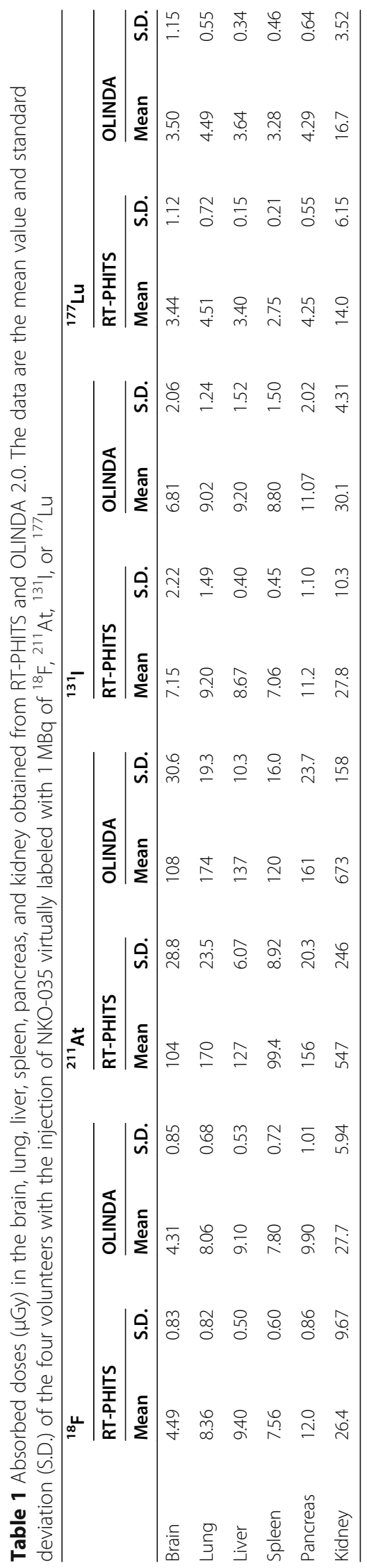




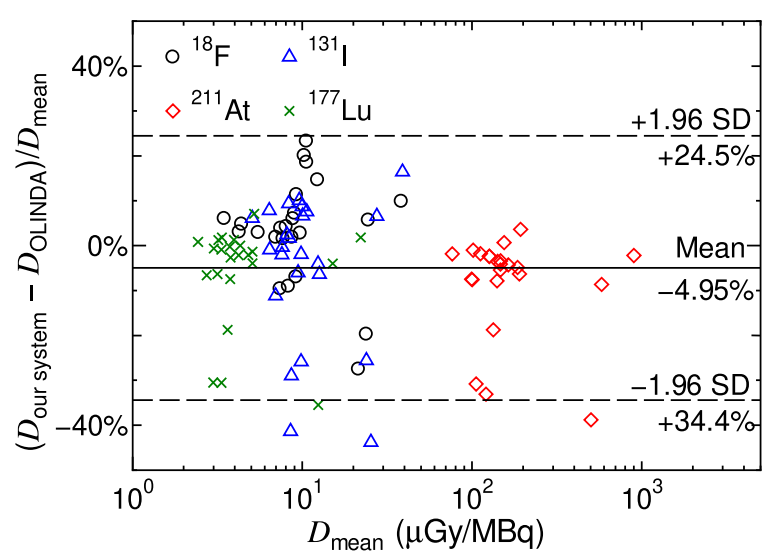

Fig. 4 Bland-Altman plot between the mean and percent difference of the organ doses calculated by RTPHITS and OLINDA 2.0 for each volunteer, radioisotope, and organ. The percent differences were obtained from ( $\left.D_{\text {RT-PHITS }}-D_{\text {OLINDA }}\right) / D_{\text {mean, }}$ where $D_{\text {RT-PHITS }}$ and $D_{\text {OLINDA }}$ are organ doses calculated by RT-PHITS and OLINDA 2.0, respectively, while $D_{\text {mean }}$ is the mean value of the two doses

\section{Discussion}

We have developed an individual dosimetry system, including the function for calculating $\operatorname{EQDX}(\alpha / \beta)$, based on PHITS coupled with the microdosimetric kinetic model. The agreements between the calculated doses obtained from RT-PHITS and OLINDA 2.0 are quite satisfactorily, confirming the reliability of our developed system. In addition, no apparent trend is observed in the Bland-Altman plot drawn in Fig. 4, suggesting that the discrepancies between RT-PHITS and OLINDA results are predominantly attributed to random issues such as anatomical differences between each volunteer and the standardized phantom adopted in OLINDA 2.0. For example, data with the percent difference out of \pm 1.96 S.D. are for organs whose masses differ from those of the standardized phantom by more than $30 \%$.

Figure 5 shows the activity dependency of the calculated dose and EQD2(4.08) in the kidney for a volunteer after injecting NKO-035 labeled with ${ }^{211}$ At or ${ }^{18} \mathrm{~F}$. The

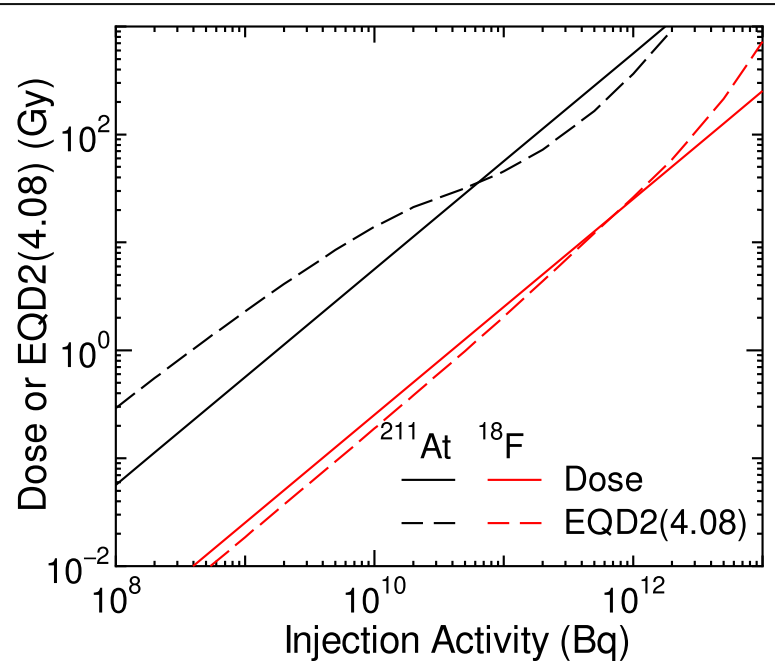

Fig. 5 Activity dependences of the calculated dose and EQD2(4.08) in the kidney for volunteer 1 with the injection of NKO-035 labeled with ${ }^{211}$ At or ${ }^{18} \mathrm{~F}$ 
calculated doses are directly proportional to the injection activity because the biokinetics of the radionuclides are assumed to be independent of their activity in this calculation. In contrast, EQD2(4.08) complicatedly depend on the injection activity. For ${ }^{211} \mathrm{At}$, they are higher and lower than the corresponding dose at lower and higher activities, respectively, and vice versa for ${ }^{18} \mathrm{~F}$.

In order to clarify these complicated relationships, we calculated EQD2(4.08) without considering the dose heterogeneity by simply averaging EQD2(4.08) in the kidney, and those without considering the dose-rate effect by setting the recovery rate constant $\mu=$ 0 . Figure 6 shows the ratios of each EQD2(4.08) to the corresponding absorbed dose as a function of the injection activity. It is evident from the graph that ignoring the dose heterogeneity results in the increase of EQD2(4.08) particularly when injecting ${ }^{211} \mathrm{At}$ with higher activities. This tendency can be explained due to the following. Firstly, the surviving fractions at high-dose irradiation are predominantly determined from those of cells having relatively smaller doses as discussed in our previous paper [33]. Lastly, the dose heterogeneity is relatively large for the injection of ${ }^{211} \mathrm{At}$ in comparison to ${ }^{18} \mathrm{~F}$, as shown in Fig. 7. Therefore, the consideration of the dose heterogeneity in a target volume is indispensable in the clinical design of TAT. The ignorance of the dose-rate effect also results in the increase of EQD2(4.08), but its influence is not so significant and is limited only at higher activities. This is because the dose rates are not very low in the studied cases owing to rather short half-lives of ${ }^{211} \mathrm{At}$ and ${ }^{18} \mathrm{~F}$, and the dose-rate effect reduces the coefficient of the quadratic term as expressed in Eq. 2, which is important only at high-dose irradiation. Note that the ratio of EQD2(4.08) to dose at lower activities becomes closer to 5.5 and 0.74 for ${ }^{211}$ At and ${ }^{18} \mathrm{~F}$, respectively, which correspond to RBE at the limit of $D \rightarrow 0, \mathrm{RBE}_{\mathrm{M}}$, multiplied with $\alpha /(\alpha+\beta X)$.

It should be mentioned that the model parameters used in these test calculations were determined from the surviving fractions of cells irradiated with external radiations, which might be inappropriate to be used for representing the surviving fraction of TAT because the absorbed doses are heterogeneously distributed in a microscopic scale due to the heterogeneity of radionuclides among each cell compartment [34] and organ microstructure [35]. Thus, the evaluation of the reliable model parameters is the

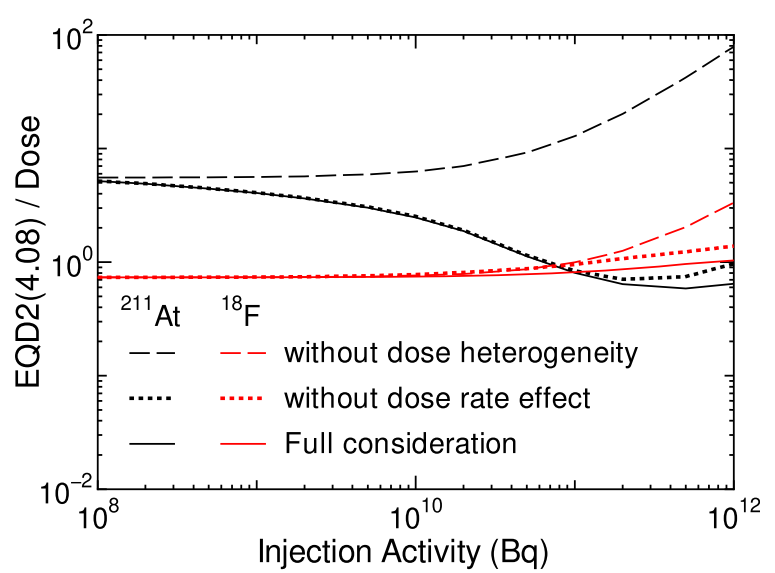

Fig. 6 Activity dependences of the ratios of EQD2(4.08) to the corresponding dose for the kidney. The data for EQD2(4.08) calculated without considering the dose heterogeneity or the dose-rate effect are also plotted 


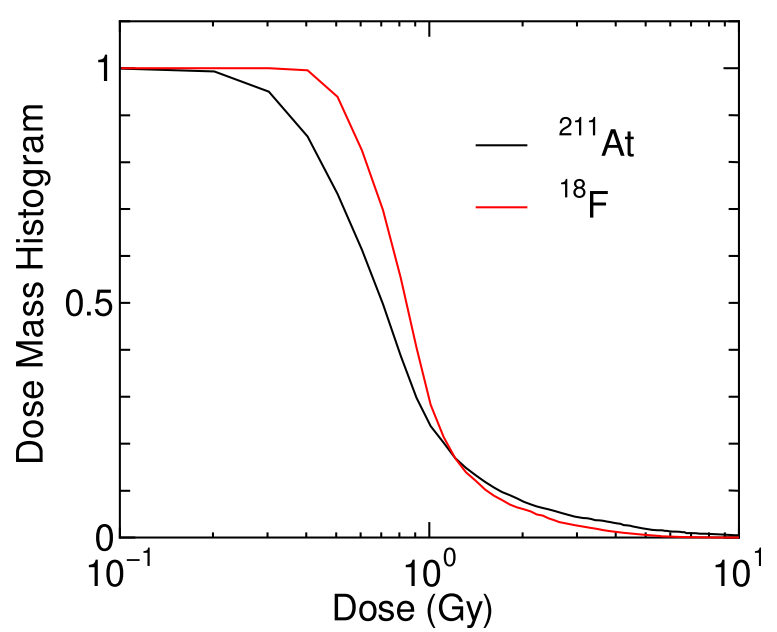

Fig. 7 Dose mass histogram (DMH) in the kidney for volunteer 1 with the injection of NKO-035 labeled with ${ }^{211}$ At or ${ }^{18} \mathrm{~F}$. The mean doses were adjusted to $1 \mathrm{~Gy}$ for both $\mathrm{DMH}$

key issue for introducing RT-PHITS in the preclinical study of TAT. For precisely calculating the doses in organs with fine structure such as stomach wall, implementation of tetrahedral-mesh phantoms in RT-PHITS is ongoing by introducing the technology developed by another PHITS-based internal dosimetry tool PARaDIM [36]. Reduction of the computational time is also desirable before the practical use of RT-PHITS in the clinic because PET-CT data of patients are generally confidential and not able to be transferred to a high-performance computer that is publicly accessible. Currently, the conventional organ dose calculation using RT-PHITS costs less than a few CPU hours, but the precise estimation of EQDX requires at least $100 \mathrm{CPU}$ hours because the statistical uncertainties in each voxel must be very small in the calculation.

\section{Conclusion}

As an extension of RT-PHITS, we developed an individual dosimetry system dedicated to nuclear medicine particularly to TAT based on PHITS coupled with the microdosimetric kinetic model. It calculates not only absorbed doses but also $\operatorname{EQDX}(\alpha / \beta)$ from the PET-CT images, considering the dose dependence of RBE, the dose-rate effect, and the dose heterogeneity. With these functionalities, RT-PHITS enables us to predict the therapeutic and side effects of TAT based on the clinical data largely available from conventional external radiotherapy. RT-PHITS including the modules developed in this study has been implemented in the latest version of PHITS, which is freely available upon the request to Japan Atomic Energy Agency.

\section{Appendix A}

Procedure for determining the cumulative activities and the biological decay constants In general, the cumulative activities are estimated by integrating the time-activity curve determined from a mono-exponential or bi-exponential fitting of the PET/SPECT images [37]. However, such fitting procedures are occasionally failed particularly when the statistical fluctuation of the measured activities was high or the number of the time steps of PET/SPECT images was small. We therefore employed a simple method for determining the decay-corrected activities, $A(t)$, by linearly interpolating the 
corresponding data obtained from the $i$ th measurement of PET/SPECT, $A_{i}$, and by extrapolating the last data, $A_{n}$, under the assumption of the mono-exponential decay, as written below:

$$
\begin{aligned}
& A(t)=a_{i} t+b_{i}=\frac{A_{i}-A_{i-1}}{t_{i}-t_{i-1}} t+\frac{A_{i-1} t_{i}-A_{i} t_{i-1}}{t_{i}-t_{i-1}} \text { for } t_{i-1}<t \leq t_{i}(i=1 \ldots n) \\
& A(t)=A_{n} e^{-\lambda_{\text {bio }}\left(t-t_{n}\right)} \text { for } t>t_{n},
\end{aligned}
$$

where $t_{i}$ is the reference time of the $i^{\text {th }}$ measurement, and $\lambda_{\text {bio }}$ represents the decay constant of the radiopharmaceutical due to the biological clearance. Note that we assumed $A_{0}=t_{0}=0$ in this calculation. The numerical value of $\lambda_{\text {bio }}$ is determined from the least-square fitting of $A_{i}$ by the mono-exponential function, excluding the data before the peak or below a certain threshold value. The fitting is regarded to be failed in the case that the fitted decay constant is negative. The actual value of $\lambda_{\text {bio }}$ used in Eq. 8 as well as Eq. 5 is calculated by averaging the fitted decay constants of 10 nearby voxels where the fitting was succeeded. Figure 8 shows an example of the decay-corrected activities obtained from Eqs. 7 and 8 in comparison with the measured data.

The cumulative activity, $C$, can be mathematically derived from $A(t)$ as follows:

$$
\begin{aligned}
C & =\frac{\lambda_{\text {phy }}}{\lambda_{\text {phy }, \text { PET }}} \int_{0}^{\infty} A(t) e^{-\lambda_{\text {phy }} t} \mathrm{~d} t \\
& =\frac{\lambda_{\text {phy }}}{\lambda_{\text {phy }, \mathrm{PET}}}\left[\sum_{i=1}^{n} \int_{t_{i-1}}^{t_{i}}\left(a_{i} t+b_{i}\right) e^{-\lambda_{\text {phy }} t} \mathrm{~d} t+A_{n} e^{-\lambda_{\text {phy }} t_{n}} \int_{t_{n}}^{\infty} e^{-\left(\lambda_{\text {phy }}+\lambda_{\text {bio }}\right)\left(t-t_{n}\right)} \mathrm{d} t\right] \\
& =\frac{\lambda_{\text {phy }}}{\lambda_{\text {phy }, \text { PET }}}\left[\sum_{i=1}^{n} \frac{\left(\lambda_{\text {phy }} t_{i-1}+1\right) e^{-\lambda_{\text {phy }} t_{i-1}}-\left(\lambda_{\text {phy }} t_{i}+1\right) e^{-\lambda_{\text {phy }} t_{i}}}{\lambda_{\text {phy }}^{2}} a_{i}+\frac{e^{-\lambda_{\text {phy }} t_{i-1}}-e^{-\lambda_{\text {phy }} t_{i}}}{\lambda_{\text {phy }}} b_{i}+\frac{A_{n} e^{-\lambda_{\text {phy }} t_{n}}}{\left(\lambda_{\text {phy }}+\lambda_{\text {bio }}\right)}\right] .
\end{aligned}
$$

where $\lambda_{\text {phy }}$ and $\lambda_{\text {phy,PET }}$ is the physical decay constants of the radionuclides used for TRT and PET/SPECT, respectively.

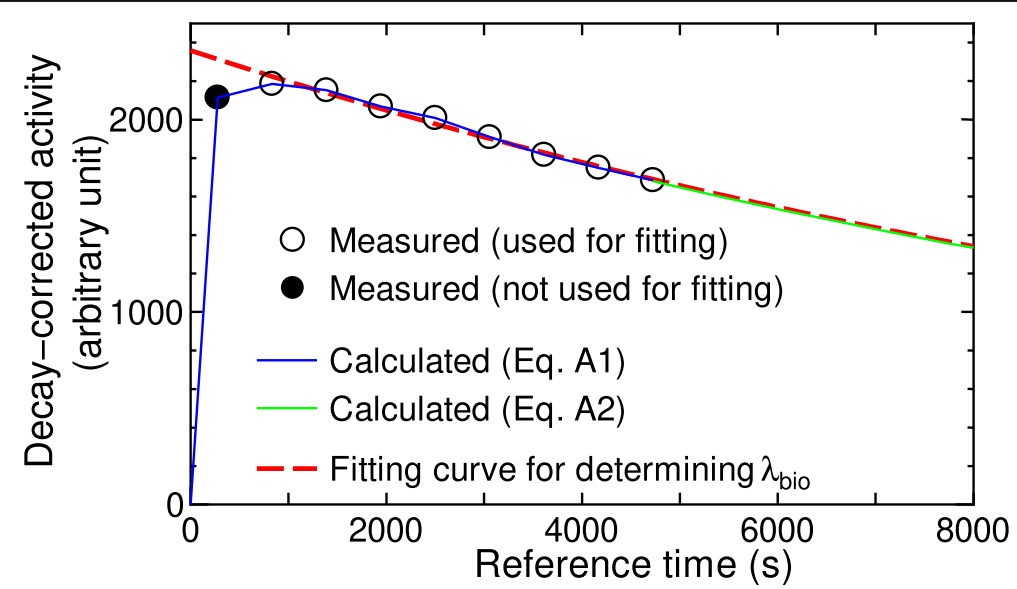

Fig. 8 Example of the decay-corrected activities calculated from Eqs. 7 and 8 in comparison with the measured data. The fitting curve for determining $\lambda_{\text {bio }}$ is also depicted 


\section{Appendix B}

Definition of fundamental microdosimetric quantities and basic features of MKM

The most important feature of microdosimetry in comparison to the conventionalscale dosimetry is the consideration of the spatial and stochastic divergences of deposition energies around the trajectories of charged particles. Thus, two stochastic quantities specially used in microdosimetry, i.e., specific energy $z$ in Gy and lineal energy $y$ in $\mathrm{keV} / \mu \mathrm{m}$, were defined and measured instead of the corresponding non-stochastic quantities used in conventional dosimetry, i.e. absorbed dose and linear energy transfer (LET), respectively. The definitions $z$ and $y$ are as follows:

$$
\begin{aligned}
& z=\frac{\varepsilon}{m}, \\
& y=\frac{\varepsilon}{\bar{l}},
\end{aligned}
$$

where $\varepsilon$ is the energy imparted to a target with mass $m$ and mean chord length $\bar{l}$. They are generally expressed in their frequency or dose probability density functions, $f(z)$ and $f(y)$ or $d(z)$ and $d(y)$, respectively. Detailed descriptions on the definition of the microdosimetric quantities are given in International Commission on Radiation Units and Measurements (ICRU) Report 36 [38].

MKM [20] is one of the most successful models to explain the biological effectiveness for the cellular surviving fraction. It mathematically interprets the LQ relation of the surviving fraction based on the theory of dual radiation action [39]. The concept of MKM is schematically drawn in Fig. 9. In MKM, the following six basic assumptions were made: (i) a cell nucleus can be divided into multiple domains with submicron

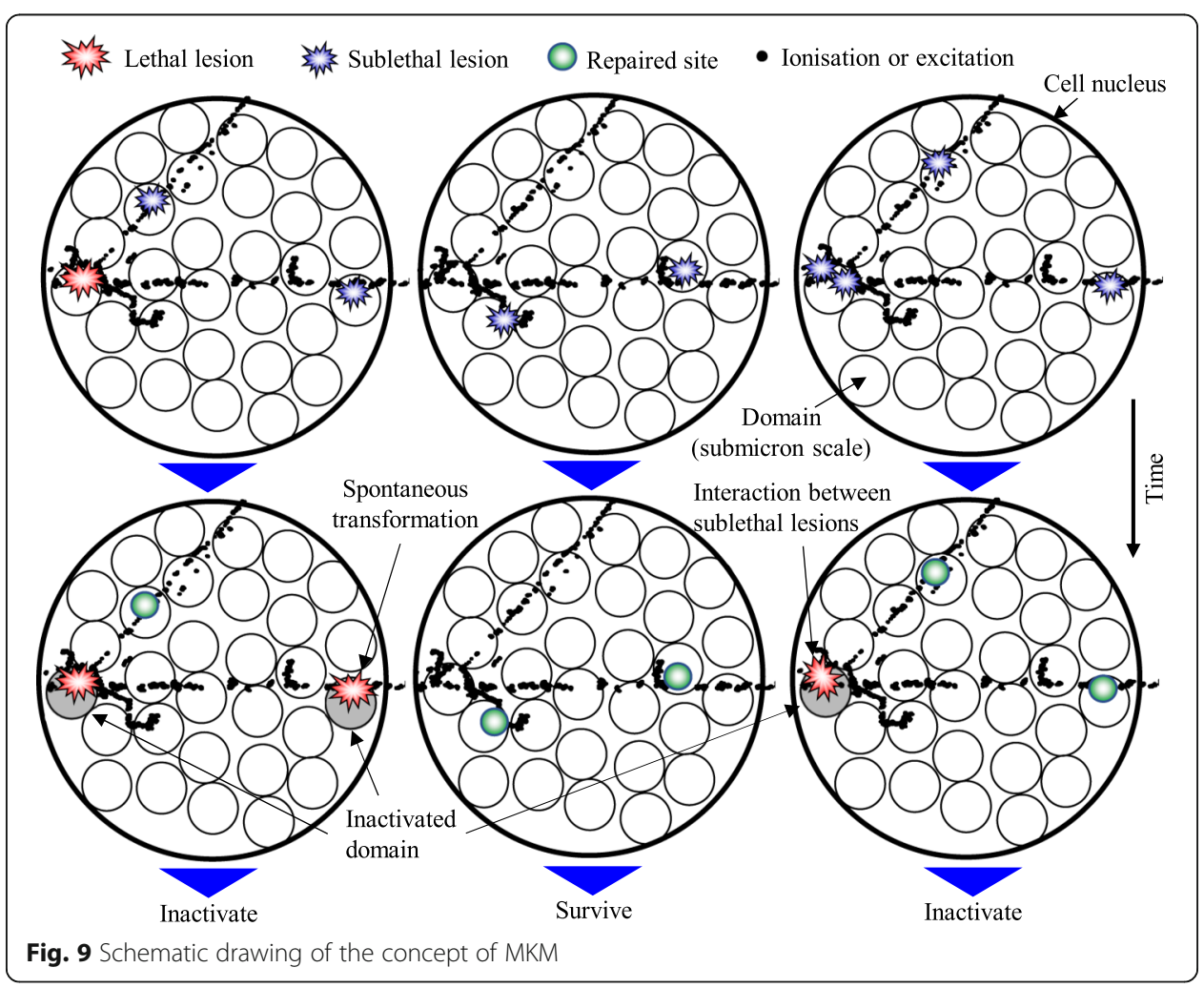


scales; (ii) radiation exposure produces two types of DNA damage named lethal and sublethal lesions in cell nuclei; (iii) the number of lethal and sublethal lesions produced in a domain is proportional to the specific energy, $z$, in the domain; (iv) a sublethal lesion is to be repaired, or converted into a lethal lesion via spontaneous transformation or interaction with another sublethal lesion created in the same domain; (v) a domain is to be considered inactivated when an intra-domain lethal lesion is formed; and (vi) a cell is to be considered inactivated when an intranuclear domain is inactivated.

Based on these assumptions, it can be mathematically derived that the cell surviving fraction in any radiation field with an absorbed dose $D, S_{\mathrm{MK}}(D)$, is calculated by

$$
S_{\mathrm{MK}}(D)=\exp \left[-\left(\alpha_{0}+\beta \bar{z}_{1 \mathrm{D}}\right) D-\beta D^{2}\right]
$$

where $\alpha_{0}$ and $\beta$ are the parameters independent of the radiation field, and $\bar{z}_{1 \mathrm{D}}$ denotes the dose-mean specific energy per event in domain. Considering the saturation correction due to the overkill effect [28] and the dose rate effect [29], Eq. 12 can be replaced by Eq. 2 .

The most important feature of MKM in comparison to other conventional LQ models is that it can computationally determine the $\alpha$ parameter for any radiation field considering the spatial and stochastic divergences of the deposition energies, owing to the use of the microdosimetric quantities $z$ and $y$ instead of LET. Thus, the RBE values of low-energy He ions calculated by MKM are higher than the corresponding data for high-energy $\mathrm{C}$ ions having the same LET, as expected from the track structure simulation and experimental data [31]. In addition, MKM suggests that the $\beta$ parameter should be independent of the radiation field, while conventional LQ models regard it as a variable and generally presume the value to be 0 for high-LET radiation. Thus, RBE obtained from by MKM are always greater than 1 in contrast to those calculated by conventional LQ models, which are occasionally less than 1 in the case of high-dose and high-LET irradiations with $\beta=0$. These features are beneficial in the estimate of RBE for TAT.

\section{Appendix C}

\section{Procedure for PET-CT data acquisition}

Whole body PET/CT images were acquired using SET-3000BCT/X, (SHIMADZU, Kyoto, Japan) in 3-D mode (pixel size $4.0 \mathrm{~mm}$, slice thickness $3.25 \mathrm{~mm}$ ) with 9 min per frame (from the mid-thigh to top-skull). PET images were reconstructed by Dynamic Row-Action Maximum Likelihood Algorithm (DRAMA) with an image matrix of $128 \times$ 128 , and a voxel size of $4.0 \times 4.0 \times 3.25 \mathrm{~mm}^{3}$. Attenuation correction was performed using

${ }^{137} \mathrm{Cs}$ source. The unenhanced low-dose CT was acquired after PET scan $(120 \mathrm{kVp}$ and $37.5 \mathrm{mAs})$. The CT-scans were reconstructed to a slice thickness of $5 \mathrm{~mm}$.

\section{Abbreviations}

BED: Biological effective dose; DMH: Dose-mass histgram; EQD: Equieffective dose; EUD: Equivalent uniform dose:

LET: Linear energy transfer; MKM: Microdosimetric kinetic model; PD: Probability density; PHITS: Particle and Heavy lon

Transport code System; RBE: Relative biological effectiveness; RT-PHITS: Radiotherapy package based on PHITS;

TAT: Targeted alpha therapy; TRT: Targeted radionuclide therapy; VOI: Volume of interest 


\section{Authors' contributions}

TS and TW contributed to the study conception and design. Calculations and code development were performed by TS, TF, and YL. PET-CT measurements and drug development were performed by SN, SN, YK, and TW. The first draft of the manuscript was written by TS. All authors read and approved the final manuscript.

\section{Funding}

PET measurement was jointly funded by J-Pharma Co. This study was funded by the QiSS program of OPERA from the Japan Science and Technology Agency (JST), Japan (Grant number: JPMJOP1721).

\section{Availability of data and materials}

The datasets used and/or analyzed during the current study are available from the corresponding author on reasonable request.

\section{Ethics approval and consent to participate}

All procedures performed in studies involving human participants were in accordance with the ethical standards of the Osaka University institutional review board and with the 1964 Helsinki declaration and its later amendments or comparable ethical standards.

\section{Consent for publication}

Informed consent was obtained from all individual participants included in the study.

\section{Competing interests}

There is no other potential conflict of interest relevant to this article to disclose.

\section{Author details}

${ }^{1}$ Nuclear Science and Engineering Center, Japan Atomic Energy Agency, Shirakata 2-4, Tokai, Ibaraki 319-1195, Japan. ${ }^{2}$ Research Center for Nuclear Physics, Osaka University, Suita, Japan. ${ }^{3}$ Department of Nuclear Medicine and Tracer Kinetics, Graduate School of Medicine, Osaka University, Suita, Japan. ${ }^{4}$ Department of Radiology, Osaka University Hospital, Suita, Japan. ${ }^{5}$ Department of Laboratory Medicine, The Jikei University School of Medicine, Tokyo, Japan. ${ }^{6}$ Department of Bio-system Pharmacology, Graduate School of Medicine, Osaka University, Suita, Japan.

Received: 27 September 2020 Accepted: 21 December 2020

Published online: 12 January 2021

\section{References}

1. Kratochwil C, Bruchertseifer F, Giesel FL, Weis M, Verburg FA, Mottaghy F, et al. 225Ac-PSMA-617 for PSMA-targeted alpha-radiation therapy of metastatic castration-resistant prostate cancer. J Nucl Med. 2016;57:1941-4. https://doi.org/10. 2967/jnumed.116.178673.

2. Watabe T, Kaneda-Nakashima K, Liu Y, Shirakami Y, Ooe K, Toyoshima A, et al. Enhancement of 211At uptake via the sodium iodide symporter by the addition of ascorbic acid in targeted alpha-therapy of thyroid cancer. J Nucl Med. 2019;60:1301-7. https://doi.org/10.2967/jnumed.118.222638.

3. Watabe T, Kaneda-Nakashima K, Shirakami Y, Liu Y, Ooe K, Teramoto T, et al. Targeted alpha therapy using astatine (211At)-labeled phenylalanine: A preclinical study in glioma bearing mice. Oncotarget. 2020;11:1388-98. https://doi.org/ 10.18632/oncotarget.27552.

4. Watabe T, Liu Y, Kaneda-Nakashima K, Shirakami Y, Lindner T, Ooe K, et al. Theranostics targeting fibroblast activation protein in the tumor stroma: 64Cu- and 225Ac-labeled FAPI-04 in pancreatic cancer xenograft mouse models. J Nucl Med. 2020;61:563-9. https://doi.org/10.2967/jnumed.119.233122.

5. Sgouros G, Roeske JC, McDevitt MR, Palm S, Allen BJ, Fisher DR, et al. MIRD Pamphlet No. 22 (abridged): radiobiology and dosimetry of alpha-particle emitters for targeted radionuclide therapy. J Nucl Med. 2010;51:311-28. https://doi.org/ 10.2967/jnumed.108.058651.

6. Karger CP, Peschke P. RBE and related modeling in carbon-ion therapy. Phys Med Biol. 2017;63:01TR2. https://doi.org/10. 1088/1361-6560/aa9102.

7. Hobbs RF, Howell RW, Song H, Baechler S, Sgouros G. Redefining relative biological effectiveness in the context of the EQDX formalism: implications for alpha-particle emitter therapy. Radiat Res. 2014;181:90-8. https://doi.org/10.1667/ RR13483.1

8. Bentzen SM, Dorr W, Gahbauer R, Howell RW, Joiner MC, Jones B, et al. Bioeffect modeling and equieffective dose concepts in radiation oncology--terminology, quantities and units. Radiotherapy Oncol. 2012;105:266-8. https://doi.org/ 10.1016/j.radonc.2012.10.006.

9. Barendsen GW. Dose fractionation, dose rate and iso-effect relationships for normal tissue responses. Int J Radiat Oncol Biol Phys. 1982;8:1981-97. https://doi.org/10.1016/0360-3016(82)90459-x.

10. Stabin MG, Sparks RB, Crowe E. OLINDA/EXM: the second-generation personal computer software for internal dose assessment in nuclear medicine. J Nucl Med. 2005;46:1023-7.

11. Andersson M, Johansson L, Eckerman K, Mattsson S. IDAC-Dose 2.1, an internal dosimetry program for diagnostic nuclear medicine based on the ICRP adult reference voxel phantoms. EJNMMI Res. 2017;7:88. https://doi.org/10.1186/ s13550-017-0339-3.

12. Kolbert KS, Sgouros G, Scott AM, Bronstein JE, Malane RA, Zhang J, et al. Implementation and evaluation of patientspecific three-dimensional internal dosimetry. J Nucl Med. 1997;38:301-8.

13. Prideaux AR, Song H, Hobbs RF, He B, Frey EC, Ladenson PW, et al. Three-dimensional radiobiologic dosimetry: application of radiobiologic modeling to patient-specific 3-dimensional imaging-based internal dosimetry. J Nucl Med. 2007:48:1008-16. https://doi.org/10.2967/jnumed.106.038000. 
14. Botta F, Mairani A, Hobbs RF, Vergara Gil A, Pacilio M, Parodi K, et al. Use of the FLUKA Monte Carlo code for 3D patient-specific dosimetry on PET-CT and SPECT-CT images. Phys Med Biol. 2013;58:8099-120. https://doi.org/10.1088/ 0031-9155/58/22/8099.

15. Marcatili S, Pettinato C, Daniels S, Lewis G, Edwards P, Fanti S, et al. Development and validation of RAYDOSE: a Geant4based application for molecular radiotherapy. Phys Med Biol. 2013;58:2491-508. https://doi.org/10.1088/0031-9155/58/8/ 2491.

16. Kost SD, Dewaraja YK, Abramson RG, Stabin MG. VIDA: a voxel-based dosimetry method for targeted radionuclide therapy using Geant4. Cancer Biother Radiopharm. 2015;30:16-26. https://doi.org/10.1089/cbr.2014.1713.

17. Besemer AE, Yang YM, Grudzinski JJ, Hall LT, Bednarz BP. Development and validation of RAPID: a patient-specific monte carlo three-dimensional internal dosimetry platform. Cancer Biother Radiopharm. 2018;33:155-65. https://doi.org/10. 1089/cbr.2018.2451.

18. Ljungberg M, Gleisner KS. 3-D image-based dosimetry in radionuclide therapy. IEEE TRPMS. 2018;2:527-40.

19. Sato T, Iwamoto Y, Hashimoto S, Ogawa T, Furuta T, Abe S, et al. Features of particle and heavy ion transport code system PHITS Version 3.02. J Nucl Sci Technol. 2018;55:684-90. https://doi.org/10.1080/00223131.2017.1419890.

20. Hawkins RB. A microdosimetric-kinetic model of cell death from exposure to ionizing radiation of any LET, with experimental and clinical applications. Int J Radiat Biol. 1996:69:739-55. https://doi.org/10.1080/095530096145481.

21. Takada K, Sato T, Kumada H, Koketsu J, Takei H, Sakurai H, et al. Validation of the physical and RBE-weighted dose estimator based on PHITS coupled with a microdosimetric kinetic model for proton therapy. J Radiat Res. 2018;59:91-9. https://doi.org/10.1093/jrr/rrx057.

22. Sato T, Kase Y, Watanabe R, Niita K, Sihver L. Biological dose estimation for charged-particle therapy using an improved PHITS code coupled with a microdosimetric kinetic model. Radiat Res. 2009;171:107-17. https://doi.org/10.1667/Rr1510. 1.

23. Sato T, Masunaga S, Kumada H, Hamada N. Microdosimetric modeling of biological effectiveness for boron neutron capture therapy considering intra- and intercellular heterogeneity in ${ }^{10} \mathrm{~B}$ distribution. Sci Rep. 2018;8:988. https://doi.org/ 10.1038/s41598-017-18871-0.

24. Schneider W, Bortfeld T, Schlegel W. Correlation between CT numbers and tissue parameters needed for Monte Carlo simulations of clinical dose distributions. Phys Med Biol. 2000;45:459-78. https://doi.org/10.1088/0031-9155/45/2/314.

25. Hirayama H, Namito Y, Bielajew AF, Wilderman SJ, Nelson WR, SLAC national accelerator laboratory and high energy accelerator research organization. The EGS5 code system. SLAC-R-730 and KEK Report 2005-8; 2005.

26. International Commission on Radiological Protection. Nuclear decay data for dosimetric calculations, ICRP Publication 107, Ann. ICRP 38(3); 2008.

27. Sato T, Watanabe R, Niita K. Development of a calculation method for estimating specific energy distribution in complex radiation fields. Radiat Prot Dosim. 2006;122:41-5.

28. Kase Y, Kanai T, Matsumoto Y, Furusawa Y, Okamoto H, Asaba T, et al. Microdosimetric measurements and estimation of human cell survival for heavy-ion beams. Radiat Res. 2006;166:629-38. https://doi.org/10.1667/RR0536.1.

29. Matsuya Y, McMahon SJ, Tsutsumi K, Sasaki K, Okuyama G, Yoshii Y, et al. Investigation of dose-rate effects and cell-cycle distribution under protracted exposure to ionizing radiation for various dose-rates. Sci Rep. 2018;8:8287. https://doi.org/ 10.1038/s41598-018-26556-5.

30. Sato T, Watanabe R, Kase Y, Tsuruoka C, Suzuki M, Furusawa Y, et al. Analysis of cell-survival fractions for heavy-ion irradiations based on microdosimetric kinetic model implemented in the particle and heavy ion transport code system. Radiat Prot Dosim. 2011;143:491-6. https://doi.org/10.1093/Rpd/Ncq484.

31. Furusawa $Y$, Fukutsu K, Aoki M, Itsukaichi H, Eguchi-Kasai K, Ohara H, et al. Inactivation of aerobic and hypoxic cells from three different cell lines by accelerated He-3-,C-12- and Ne-20-ion beams. Radiat Res. 2000;154:485-96. https://doi.org/ 10.1667/0033-7587(2000)154[0485:Ioaahc]2.0.Co;2.

32. O'Donoghue JA. Implications of nonuniform tumor doses for radioimmunotherapy. J Nucl Med. 1999;40:1337-41.

33. Sato T, Furusawa Y. Cell survival fraction estimation based on the probability densities of domain and cell nucleus specific energies using improved microdosimetric kinetic models. Radiat Res. 2012;178:341-56. https://doi.org/10.1667/ Rr2842.1.

34. Goddu SM, Howell RW, Rao DV. Cellular Dosimetry - Absorbed fractions for monoenergetic electron and alpha-particle sources and S-values for radionuclides uniformly distributed in different cell compartments. J Nucl Med. 1994;35:303-16.

35. Hobbs RF, Song H, Huso DL, Sundel MH, Sgouros G. A nephron-based model of the kidneys for macro-to-micro alphaparticle dosimetry. Phys Med Biol. 2012;57:4403-24. https://doi.org/10.1088/0031-9155/57/13/4403.

36. Carter LM, Crawford TM, Sato T, Furuta T, Choi C, Kim CH, et al. PARaDIM: A PHITS-based Monte Carlo tool for internal dosimetry with tetrahedral mesh computational phantoms. J Nucl Med. 2019;60:1802-11. https://doi.org/10.2967/ jnumed.119.229013.

37. Sarrut D, Halty A, Badel JN, Ferrer L, Bardies M. Voxel-based multimodel fitting method for modeling time activity curves in SPECT images. Med Phys. 2017:44:6280-8. https://doi.org/10.1002/mp.12586.

38. International Commission on Radiation Units and Measurements. Microdosimetry. ICRU Report, vol. 36; 1983.

39. Kellerer AM, Rossi HH. A generalized formation of dual radiation action. Radiat Res. 1978;75:471-88.

\section{Publisher's Note}

Springer Nature remains neutral with regard to jurisdictional claims in published maps and institutional affiliations. 